\title{
Realization and characterization of XUV multilayer coatings for attosecond pulses
}

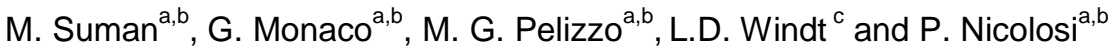 \\ a. CNR - National Institute for the Physics of the Matter, LUXOR Laboratory, Italy \\ b. Information Engineering Department, University of Padova, Italy; c. Reflective X-ray Optics \\ LLC, New York, USA \\ mailto:sumanmici@dei.unipdt.it
}

\begin{abstract}
Multilayer techniques are used to obtain high reflectivity in the XUV spectral region. This work presents the realization of aperiodic multilayer mirrors for attosecond pulse compression and their characterization through reflectivity and photoemission spectroscopy measurements.
\end{abstract}

\section{Introduction}

High Order Harmonics are generated by ionization of atoms with ultra-intense and ultra-short infrared LASER pulses. Harmonics spectrum bandwidth covers a wide EUV spectral region allowing the generation of attosecond pulses by amplitude and phase modification of each spectral component. Generation of attosecond pulses can be realized by filtering a particular spectral region, moreover, additional time compression can be obtained by the harmonics phase reordering.

The realization of broadband multilayers able to reflect and compress attosecond pulses is a crucial point for attosecond research. We have realized multilayers with broadband rectangular reflectivity and negatively chirped phase trend able to comply the high order harmonics phase mismatch. The aperiodic multilayers has been designed by a suitably developed optimization algorithm [1].

We show the experimental characterization of a Mo/Si broadband multilayer designed for compressing attosecond pulses in the EUV region, they have been characterized at the BEAR beamline of ELETTRA synchrotron. The multilayer phase characterization has been derived using an innovative method based on the total electron yield signal [2].

\section{$2 \mathrm{ML}$ structure design}

The design of aperiodic multilayers has been derived with a suitably developed optimization algorithm [1].

Since the optimal reflection behavior depends strongly on the multilayer structure the optimization procedure is based on an algorithm with evolutive strategy. The algorithm implementation takes into accounts inter-reflections between different layers and interdiffusion at the interfaces. The procedure has been structured to find aperiodic multilayers with stable performance with varying layer dimensions [3], in order to assure the reliability of the optimized solution. S/W has been developed in the matlab® language and interactive environment.

\section{Phase characterization through photoemission signal}

A multilayer designed in order to reflect attosecond pulses using the method described in the previous section has been deposited at RXOLLC [4] and characterized through reflectivity and phase measurements at BEAR beamline [5]. In particular the phase of the multilayer has been recovered from Total Emission Yield measurements and the results obtained are discussed. Differently from others [6], the TEY method proposed in this paper should have an important advantage: it should be insensitive to the materials irregularity present into the multilayer surface due to oxidation or carbon film formation on top of the ML. The proposed method will overcome this problem by 
elaboration of TEY signals coming from the sample under test instead of using an external sample as a reference (as Si film), removing the error due to the different morphology of the two films. Furthermore, a second method is proposed in order to extend the measurements also to regions of absorption edges.

\section{Experimental results}

In Fig. 1 the results of the reflectivity measurements, in continuous line, compared with the theoretical prediction, in dotted line. Experimental data are shifted of few eV towards higher energy value this is due to a small drift in the deposition rate. The experimental curve match quite well the theoretically prediction. Interdiffusion and roughness at the interfaces can affect the reflectivity amplitude, while the polarization degree of incident light is only minimally diminishing such curve as previously discussed. The effect of a thin carbon layer has been simulated, resulting in a negligible effect over reflectivity.

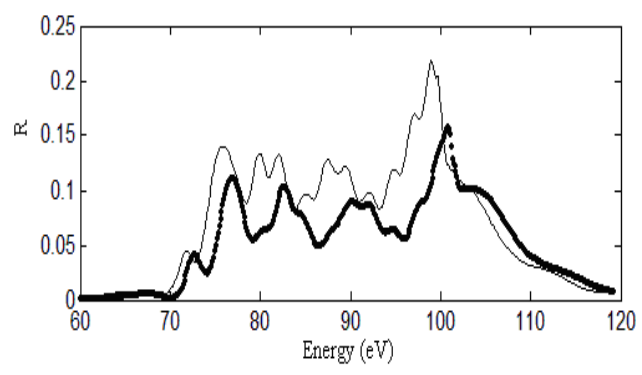

Fig. 1 Theoretical (continuous line) and experimental (dotted line) reflectivity.

The phase recovered in the whole spectral range is reported in Fig.2. Between 90 and $100 \mathrm{eV}$ both methods have been used, showing a very good agreement in the overlapped spectral range, even if the second method is more noisy. The phase difference between experimental and theoretical curves can be due to interfacial roughness (estimated in $8 \AA$ ) and also the presence of a carbon layer on top of the structure.

In conclusion we are able to design realize and characterize multilayers for the reflection and compression of attosecond pulses.

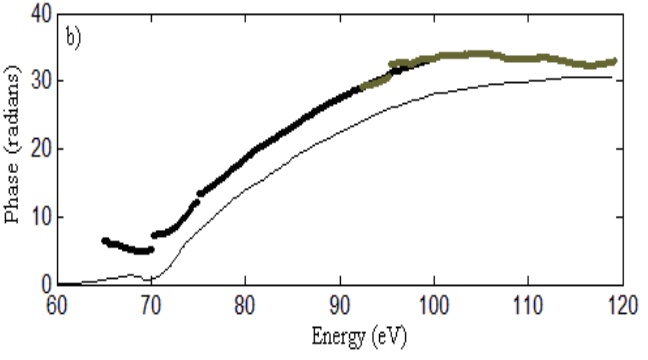

Fig.2 Phase experimentally derived by the use of method I (black) and method II (grey). In continuous line the theoretical expected.

\section{Acknowledgment}

The work has been supported by ASI grant $\mathrm{N}$. $1 / 015 / 07 / 0$ and has been èerformed within the COST Action MPO601

\section{References}

[1] Patent PCT/EP2007/060477, University of Padova, CNR-INFM, RXOLLC (N.Y. USA), 2007.

[2] Suman M. et al., "Realization and characterization of an XUV multilayer coating for attosecond pulses" in Optics Express 17: 7922-7932 (2009) and in these proceedings

[3] Suman M. et al, in Applied Optics 46(33): 8159-8169 (2007)

[4] Windt D. L. et al. in J. Vac. Sci. Technol. B 12, 3826-3832 (1994)

[5] http://www.elettratrieste.it/experiments/ beamlines/bear

[6] A. Aquila et al. Opt. Lett. 33(5), 2008. 